\title{
Video Article \\ Development of Obliterative Bronchiolitis in a Murine Model of Orthotopic Lung Transplantation
}

\author{
Hidemi Suzuki ${ }^{1,2}$, Lin Fan ${ }^{1,2}$, David S. Wilkes ${ }^{1,2}$ \\ ${ }^{1}$ Departments of Medicine, Microbiology and Immunology, Indiana University School of Medicine \\ ${ }^{2}$ Center for Immunobiology, Indiana University School of Medicine
}

Correspondence to: David S. Wilkes at dwilkes@iupui.edu

URL: https://www.jove.com/video/3947

DOI: doi:10.3791/3947

Keywords: Medicine, Issue 65, Immunology, Microbiology, Physiology, lung, transplantation, mouse, obliterative bronchiolitis, vascularized lung transplants

Date Published: 7/10/2012

Citation: Suzuki, H., Fan, L., Wilkes, D.S. Development of Obliterative Bronchiolitis in a Murine Model of Orthotopic Lung Transplantation. J. Vis. Exp. (65), e3947, doi:10.3791/3947 (2012).

\section{Abstract}

Orthotopic lung transplantation in rats was first reported by Asimacopoulos and colleagues in $1971^{1}$. Currently, this method is well accepted and standardized not only for the study of allo-rejection but also between syngeneic strains for examining mechanisms of ischemia-reperfusion

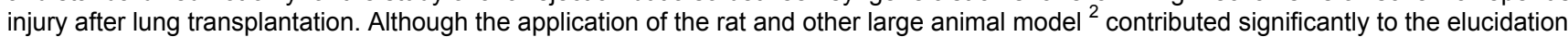
of these studies, the scope of those investigations is limited by the scarcity of knockout and transgenic rats. Due to no effective therapies for obliterative bronchiolitis, the leading cause of death in lung transplant patients, there has been an intensive search for pre-clinical models that replicate obliterative bronchiolitis. The tracheal allograft model is the most widely used and may reproduce some of the histopathologic features of obliterative bronchiolitis ${ }^{3}$. However, the lack of an intact vasculature with no connection to the recipient's conducting airways, and incomplete pathologic features of obliterative bronchiolitis limit the utility of this model ${ }^{4}$. Unlike transplantation of other solid organs, vascularized mouse lung transplants have only recently been reported by Okazaki and colleagues for the first time in $2007^{5}$. Applying the basic principles of the rat lung transplant, our lab initiated the obliterative bronchiolitis model using minor histoincompatible antigen murine orthotopic single-left lung transplants which allows the further study of obliterative bronchiolitis immunopathogenesis ${ }^{6}$.

\section{Video Link}

The video component of this article can be found at https://www.jove.com/video/3947/

\section{Donor Procedure}

1. All surgical procedures were performed utilizing sterile technique. No antibiotics are given to both donor and recipient mice.

2. Induction of anesthesia of the donor mouse is initiated with $5 \%$ Isoflurane.

3. The mouse is orotracheally intubated with a 20 -gauge intravenous catheter and then placed on a rodent ventilator, using $100 \%$ oxygen at a rate of 125 breaths/minute and approximately $0.5 \mathrm{ml}$ tidal volume ( $2 \%$ of its body weight).

4. Anesthesia is maintained with inhaled $1-2 \%$ Isoflurane.

5. The donor mouse is placed in a supine position, prepped with $70 \%$ alcohol

6. Laparosternotomy is performed as a combined midline and transverse incision.

7. Heparin at $100 \mathrm{u} / \mathrm{kg}$ is injected into the Inferior vena cava (IVC), from just below the liver.

8. The diaphragm is cut along the ventral costal attachment toward the spine, and the thoracic cavity is exposed by cutting both sides of the chest wall to the neck.

9. After incising the IVC at the level of diaphragm, the right atrial appendage is cut and the lungs flushed with $2 \mathrm{ml}$ of cooled $\left(4{ }^{\circ} \mathrm{C}\right)$ Lactated Ringer's injection and $0.1 \mathrm{ml}$ of heparin via a transverse incision at the root of the pulmonary artery (PA) trunk.

10. Arresting ventilation at two thirds of end-tidal inflation, the heart-lung block is excised and stored on ice $\left(4^{\circ} \mathrm{C}\right)$.

11. The donor left lung is prepared for the recipient by the attachment of cuffs. The pulmonary ligament is incised up to the Pulmonary Vein (PV). The hilum is brought out by removing esophagus and aorta.

12. Identify the PA, located at the most cranial aspect of the hilum, as well as the attached main bronchus (Br). Carefully dissect the PA from the bronchus.

13. PA cuff is made from a 24-gauge Intravenous (I.V.) catheter and cut to $0.5 \mathrm{~mm}$ in length with an extension of $0.7 \mathrm{~mm}$.

14. The entire surface of the cuff is then abraded to facilitate anastomosis.

15. The cuff for the bronchus is derived from a 20-gauge IV catheter, and cut in length of $1.0 \mathrm{~mm}$ with an extension of $0.7 \mathrm{~mm}$.

16. Using the same materials, the PV cuff varies with the weight of the donor mouse. Specifically, for mice $24-27$ grams the cuff size is $22-$ gauge, $0.7 \mathrm{~mm}$ in length with a $0.7 \mathrm{~mm}$ extension. For mice weighing $27-32$ grams, the studies utilized a 20 -gauge catheter that is $0.7 \mathrm{~mm}$ in length with a $0.7 \mathrm{~mm}$ extension. 
17. The cuffs are inserted into the distal ends of the PA, PV and $\mathrm{Br}$ and secured with a 9-0 suture.

18. The donor lung is flushed and washed with sterile saline with sterile heparin before storage. The donor lung is then wrapped with sterile gauze soaked in sterile saline on ice $\left(4^{\circ} \mathrm{C}\right)$, which keeps the lung very clean and sterile.

19. A microvessel clamp is placed on the bronchus to prevent Lactated Ringer entry into the airway.

\section{Recipient Procedure}

1. Induction of anesthesia and mechanical ventilation are the same as described for the donor above.

2. The left chest wall is shaved and prepped with $70 \%$ alcohol and the surgical field is draped.

3. A thoracotomy incision is made in the left third intercostal space, extending the incision dorsally close to the spine, and a microvessel clamp placed on the left pulmonary vessels and bronchus adjacent to the heart. You can see PA at the cranial aspect, PV at the caudal end of hilum and $\mathrm{Br}$ between them.

4. Using gentle traction on the hemostat to cause mild tension on the PA, Br and PV, the left lung is pulled out from the thoracic cavity while leaving the central hilar structures clamped.

5. The PA, PV and $\mathrm{Br}$ are isolated by blunt dissection followed by placing a 9-0 suture positioned loosely around the PA, PV and Br.

6. After dissecting the PA completely from its adventitial sheath, a small transverse incision of approximately one fourth of the vessel's circumference is made into the anterior wall, leaving the continuation of the dorsal part of the artery intact.

7. The donor lung, wrapped in cold, Lactated Ringer-soaked cotton gauze and prepared as described above, is then positioned into the thoracic cavity, and cuffs inserted into the recipient PA, PV and Br and secured with 9-0 suture.

8. The hilar cross clamp is removed allowing for reperfusion and ventilation.

9. After positioning the transplanted lung back into the recipient thorax, the thoracotomy incision is closed using a 5-0 suture.

10. The mouse is allowed to recover from anesthesia. Buprenorphine $(0.05-0.15 \mathrm{mg} / \mathrm{kg})$ is administered immediately after surgery, and for every 8 hours for 2-3 days post-surgery.

\section{Representative Results}

Our experience has taught us that it requires several months of repeated practice to become proficient in the mouse lung transplant model. After proficiency is attained, we achieved a $96 \%$ (96/100 consecutive surgeries) perioperative survival rate with deaths occurring within seven days post operatively. Two deaths were due to bleeding that began intraoperatively, and pneumothraces were the cause of death in the other two mice. For all procedures, the warm ischemia time was $14.32 \pm 3.14$ minutes, and cold ischemia time was $58.51 \pm 18.06$ minutes. Three orthotopic lung transplant groups were studied: isograft: C57BL/6 $\rightarrow$ C57BL/6, allograft: $\mathrm{C} 57 \mathrm{BL} / 10 \rightarrow \mathrm{C} 57 \mathrm{BL} / 6$ and C57BL/6 $\rightarrow \mathrm{C} 57 \mathrm{BL} / 10$. We used only male mice but our technique can be also applied to female mice, because there is no significant anatomical difference between the sexes.

Grading of rejection pathology was conducted in a blinded fashion utilizing standard criteria for clinical lung transplantation ${ }^{7}$ (Table 1). Whereas we observed mild or no rejection in isograft (C57BL/6 $\rightarrow$ C57BL/6), both allograft combinations developed comparable acute or chronic rejection (Figure 1). In contrast, $\mathrm{OB}$ was significantly more frequent in the $\mathrm{C} 57 \mathrm{BL} / 10 \rightarrow \mathrm{C} 57 \mathrm{BL} / 6$ than $\mathrm{C} 57 \mathrm{BL} / 6 \rightarrow \mathrm{C} 57 \mathrm{BL} / 10$ group by day28 (Table 1 ).

\begin{tabular}{|c|c|c|c|c|}
\hline & $\begin{array}{l}\mathrm{C} 57 \mathrm{BL} / 6 \rightarrow \\
\mathrm{C} 57 \mathrm{BL} / 6\end{array}$ & $\begin{array}{l}\mathrm{C} 57 \mathrm{BL} / 10 \rightarrow \\
\mathrm{C} 57 \mathrm{BL} / 6\end{array}$ & $\begin{array}{l}\mathrm{C} 57 \mathrm{BL} / 6 \rightarrow \\
\mathrm{C} 57 \mathrm{BL} / 10\end{array}$ & P-value \\
\hline $\begin{array}{l}\text { Rejection Pathology } \\
\text { "A" Scores at day } 28\end{array}$ & $0.67 \pm 0.89$ & *3.33 \pm 0.82 & *3.29 \pm 0.76 & ${ }^{*} \mathrm{P}>0.05$ \\
\hline $\begin{array}{l}\text { OB/Total mice at day } 21 \\
\text { and } 28\end{array}$ & $0 / 24(0 \%)$ & **14/34 (42.1\%) & **2/16 (12.5\%) & ${ }^{* *} P<0.05$ \\
\hline
\end{tabular}

Table 1. Histological scores of acute rejection and prevalence of obliterative bronchiolitis post transplant. Scoring of acute rejection ("A" Scores) by standard criteria as described in representative results. Data represents the mean \pm SD of "A" Scores at days 28 post transplantation. Data represents the quantity and percentage of mice in each group that developed $\mathrm{OB}$ at days 21 and 28 post transplantation. 


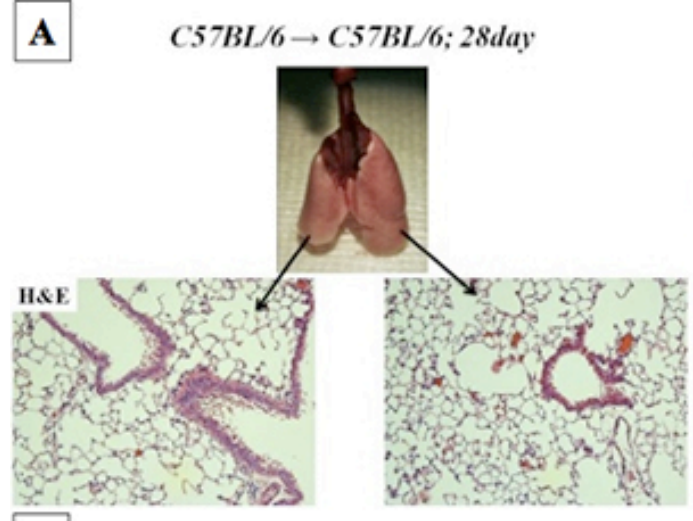

B $C 57 B L / 10 \rightarrow C 57 B L / 6 ; 28 d a y-O B$ case
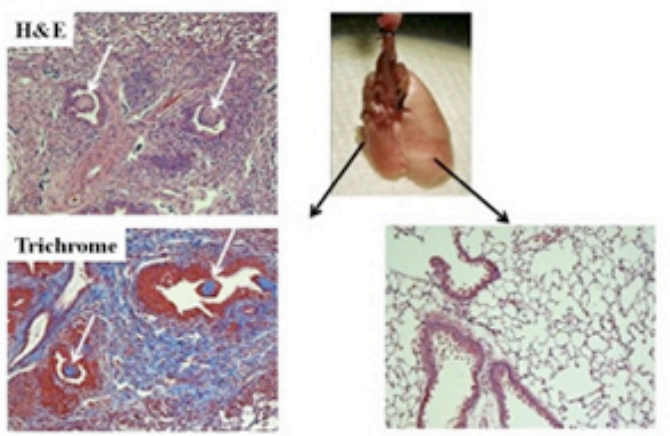

$\mathrm{C} C 57 B L / 10 \rightarrow C 57 B L / 6 ; 28$ day - non $\mathrm{OB}$ case
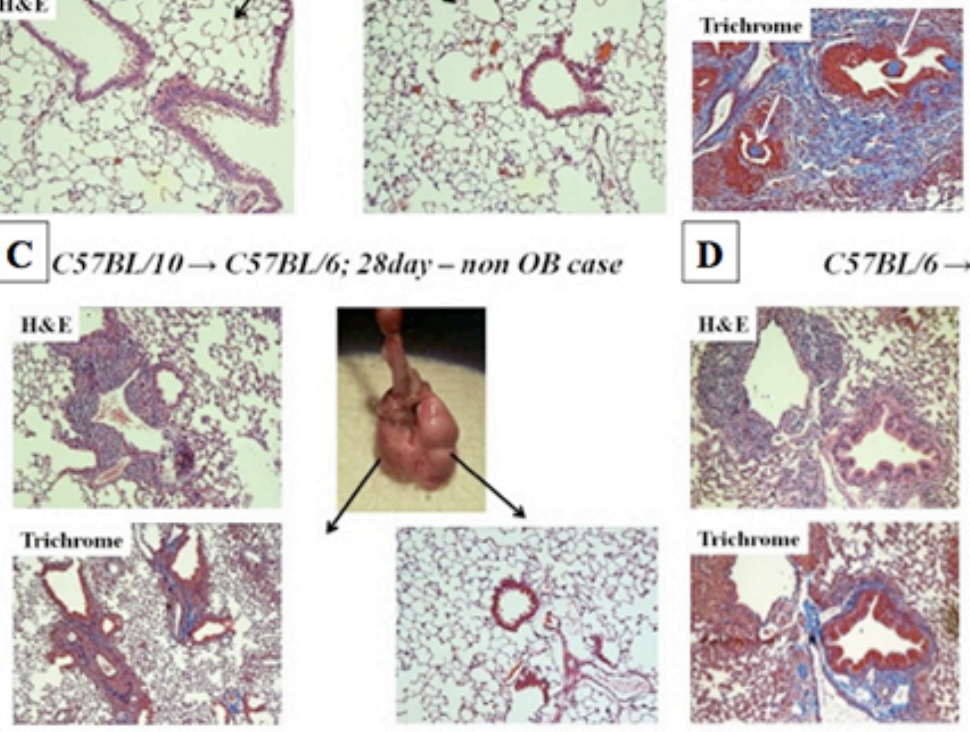

\section{D $\quad$ C57BL/6 $\rightarrow C 57 B L / 10 ; 28 d a y$}
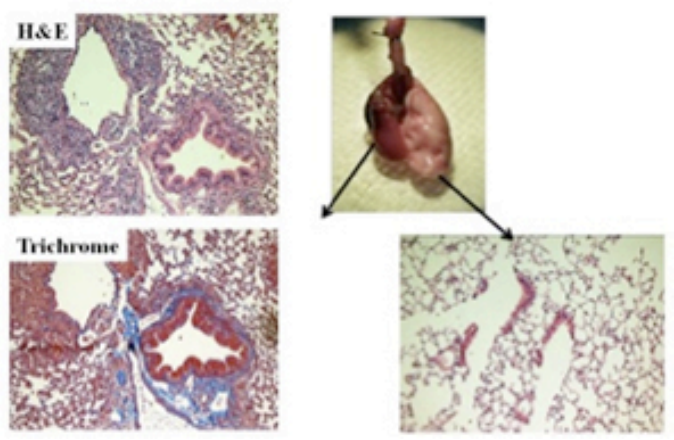

Figure 1. Macroscopic findings and histopathology at 28 days post lung transplantation. Panel $1 \mathrm{~A}$ represent macroscopic findings and $\mathrm{H} \& \mathrm{E}$ stained Isograft lung and right naïve lung. Panel $1 \mathrm{~B}$ and $1 \mathrm{C}$ represents H\&E and Masson's Trichrome stained BL/10 lung allograft transplanted into BL/6 mouse recipient, which developed OB and non OB, respectively. The white arrow in 1B identifies lesions of OB. Panel 1D shows BL/6 lung allograft transplanted into $\mathrm{BL} / 10$ mouse recipient.

Main trouble shooting during the procedure were as follows.

1. Blood flow disorder: Flush donor lung until the color becomes white in color. Use of excessive pressure could induce pulmonary edema. But do not push too much to prevent edema after transplant.

2. Difficulty of cuff insertion into the donor: Ascertain that vasculature is of sufficient length and free of attached fat and connective tissues.

3. Bleeding at anastomotic site: Use $Q$ tips to apply pressure at affected site for approximately 5 minutes.

4. Recipient PA blood flow obstruction: Mainly caused by torsion of the during cuff insertion. If air is observed within the PA then re-do of the anastomosis is necessary.

5. Difficult PV anastomosis: Membrane of PV is very thin and easily tears. Select proper cuff size and insert straight to the recipient's PV very gently.

6. Recipient PV blood flow obstruction: Make sure the position and direction of the cuff. Release the compression from the bronchial cuff.

7. Pneumothorax: This may be caused by either bronchial perforation due to manipulation of the airway, or perforation of lung surface due to trauma associated with the transplant procedure. The former is treated with surgical closure of the hole within the airway wall; and the latter is treated by oversewing the leak on the lung surface using 10-0 nylon suture.

\section{Discussion}

Orthotopic lung transplantation in mice is challenging due to microsurgical demands and extreme fragility of tissues. Introduction of the cuff technique has allowed for the widespread use of the orthotopic lung transplantation in rats ${ }^{8}$. This became the basis for the development of the orthotopic lung transplant model in mice in our lab. In mice and rats, unlike humans, the left lung contains only one lobe and makes up only $25 \%$ of the total lung mass. This makes left-single lung transplantation feasible in the murine model without the need for a circulatory support system.

Our preliminary surgeries revealed that ventilation and perfusion of the transplanted lung was highly dependent on the size of PV anastomosis. Specific PV anastomotic cuff size were utilized to match donor and recipients as reported ${ }^{9}$. Inappropriate cuff sizing resulted in either atelectasis of the transplanted lung or dehiscence of the PV anastomosis. Okazaki et al reported the use of combined vessel ligation and clipping the bronchus ${ }^{5}$. In the current study we use an aneurysm clip for all hilar structures, which we suggest may shorten warm ischemic time. One limitation of the orthotopic left lung transplant in the murine model is that recipient animals can survive after alloimmune-mediated necrosis of their allografts ${ }^{5}$. Therefore, survival studies are not feasible and graft assessment depends on histological examination of the transplanted lung ${ }^{10}$

Using this technique, we have developed a preclinical obliterative bronchiolitis model in mice. Specifically, the donor mouse is C57BL/10 and the recipient is $\mathrm{C} 57 \mathrm{BL} / 6$. This suggests the role of minor, and not major, histocompatibility antigens in obliterative bronchiolitis pathogenesis ${ }^{6}$. Furthermore, we have reported neutralizing IL-17 prevents obliterative bronchiolitis in the murine model. This model represents a novel research tool for the examination of lung transplantation and the advancement of clinical lung transplants. 


\section{Disclosures}

No conflicts of interest declared.

\section{Acknowledgements}

Funding sources: This work as supported by National Institutes of Health grants HL067177, HL096845, and P01AI084853 to D.S.W.

\section{References}

1. Asimacopoulos, P.J., Molokhia, F.A., Pegg, C.A., \& Norman, J.C. Lung transplantation in the rat. Transplant Proc. 3, 583-585, (1971)

2. Yoshida, S., et al. Surgical technique of experimental lung transplantation in rabbits. Ann. Thorac. Cardiovasc. Surg. 11, 7-11 (2005).

3. McDyer, J.F. Human and murine obliterative bronchiolitis in transplant. Proc. Am. Thorac. Soc. 4, 37-43 (2007).

4. Sato, M., Keshavjee, S., \& Liu, M. Translational research: animal models of obliterative bronchiolitis after lung transplantation. Am. J. Transplant. 9, 1981-1987 (2009).

5. Okazaki, M., et al. A mouse model of orthotopic vascularized aerated lung transplantation. Am. J. Transplant. 7, 1672-1679 (2007).

6. Fan, L., et al. Neutralizing IL-17 Prevents Obliterative Bronchiolitis in Murine Orthotopic Lung Transplantation. Am. J. Transplant. 11, 911-922 (2011).

7. Stewart, S., et al. Revision of the 1996 working formulation for the standardization of nomenclature in the diagnosis of lung rejection. J. Heart Lung Transplant. 26, 1229-1242 (2007).

8. Mizuta, T., Kawaguchi, A., Nakahara, K., \& Kawashima, Y. Simplified rat lung transplantation using a cuff technique. J. Thorac. Cardiovasc. Surg. 97, 578-581 (1989).

9. Jungraithmayr, W.M., Korom, S., Hillinger, S., \& Weder, W. A mouse model of orthotopic, single-lung transplantation. J. Thorac. Cardiovasc. Surg. 137, 486-491 (2009).

10. Li, W., et al. Orthotopic vascularized right lung transplantation in the mouse. J. Thorac. Cardiovasc. Surg., (2010). 